\title{
On growth and torsion of groups
}

\author{
Laurent Bartholdi and Floriane Pochon
}

To the memory of Narain Datt Gupta, $\dagger 11$ April 2008

\begin{abstract}
We give a subexponential upper bound and a superpolynomial lower bound on the growth function of the Fabrykowski-Gupta group.

As a consequence, we answer negatively a question by Longobardi, Maj and Rhemtulla about characterizing groups containing no free subsemigroups on two generators.
\end{abstract}

Mathematics Subject Classification (2000). 20F69, 20F50.

Keywords. Word growth, free subsemigroup.

\section{Introduction}

Fabrykowski and Gupta constructed in 1985 a group of intermediate word growth, producing in this way a new example after Grigorchuk's original construction [Gri83].

This group appears originally in [FG85], and is studied further in [FG91]; some of its algebraic properties are explained in [BG02]. A proof of its intermediate growth was first given in [FG85], with an upper bound of the form $e^{n^{\alpha}}$. The proof was not considered altogether complete, and the authors gave a second proof, in [FG91], this time with no upper bound.

In this paper, we simplify and expand somewhat this last proof, introducing the general notion of "incompressible group elements". We also derive explicit lower and upper bounds for the growth function.

Let us say that two functions $f, g$ satisfy the relation $f \lesssim g$ if there is a constant $C>0$ such that $f(n) \leq g(C n)$. We prove the

Theorem 1. The growth of the Fabrykowski-Gupta group is intermediate. More precisely, if $\gamma(n)$ denote the number of elements expressible as a product of at most $n$ generators of the Fabrykowski-Gupta group, then

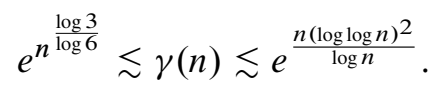


We then apply this result to a question by Longobardi, Maj and Rhemtulla. Let $G$ be a group with an exact sequence $1 \rightarrow N \rightarrow G \rightarrow P \rightarrow 1$, where $N$ is locally nilpotent and $P$ is periodic. Then $G$ has no free subsemigroup. Indeed, let $x, y \in G$. Then $x^{n}, y^{n} \in N$ for some $n$ large enough, so that $\left\langle x^{n}, y^{n}\right\rangle$ is nilpotent. Hence, neither $\left\langle x^{n}, y^{n}\right\rangle$ nor $\langle x, y\rangle$ are free as semigroups. (Note that, without loss of generality, one may assume that $G$ is finitely generated.)

In [LMR95], Longobardi, Maj and Rhemtulla asked whether the converse were true:

Question 2. Let $G$ be a finitely generated group with no free subsemigroups. Is $G$ a periodic extension of a locally nilpotent group?

The answer turns out to be negative; indeed, Ol'shanskii and Storozhev construct in [OS96] a semigroup identity whose free group is not even a periodic extension of a locally soluble group.

We remark that a very simple answer can be given to Question 2, knowing that the Fabrykowski-Gupta group has intermediate growth, and that locally nilpotent groups are elementary amenable whence have locally polynomial growth:

Theorem 3. The Fabrykowski-Gupta $G$ group is generated by two elements, contains no free subsemigroup, and is not a periodic extension of an elementary amenable group.

Proof. Consider a short exact sequence $1 \rightarrow N \rightarrow G \rightarrow P \rightarrow 1$, with $P$ periodic. Since $G$ is not periodic (as it contains the element at of infinite order), we have $N \neq 1$. Since $G$ is just infinite by Proposition 6.2 in [BG02], the index of $N$ in $G$ is finite, so $N$ is finitely generated. Therefore, since $G$ has intermediate growth, so does $N$. In particular, $N$ is not elementary amenable.

(Actually, the Fabrykowski-Gupta group has a torsion-free subgroup of index 3, see Theorem 6.4 in [BG02].)

This example is quite different from the Ol'shanskii-Storozhev example: it is a group constructed by a concrete action on a regular rooted tree (while Ol'shanskiiStorozhev's group is constructed by its presentation); accordingly, much more information can be gathered on $G$, for instance that it is a residually-3 group which does not satisfy any identity.

\section{Settings}

2.1. The Fabrykowski-Gupta group. Consider the cyclic group of order three $A=\mathbb{Z} / 3 \mathbb{Z}=\{0,1,2\}$ with generator $a$, and the 3 -regular rooted tree $\mathcal{T}_{3}=A^{*}$, with root $\emptyset$. We shall write $A^{\times}=\{1,2\}$. The automorphism group of $A^{*}$ is recursively 
defined by $\operatorname{Aut}\left(A^{*}\right)=\operatorname{Aut}\left(A^{*}\right) \prec \operatorname{Sym}(A)$, and every automorphism decomposes via the map

$$
\phi: \operatorname{Aut}\left(A^{*}\right) \rightarrow \operatorname{Aut}\left(A^{*}\right) \gtrless \operatorname{Sym}(A) ; \quad f \mapsto\left\langle\left\langle f_{0}, f_{1}, f_{2}\right\rangle\right\rangle \sigma
$$

where $f_{i} \in A^{*}$ and $\sigma \in \operatorname{Sym}(A)$. Thus, $a$ acts on $\mathcal{T}_{3}$ as a cyclic permutation of the first level $A$ of the tree. Define the automorphism $t$ recursively by $t=\langle\langle a, 1, t\rangle\rangle$. Note that both $a$ and $t$ are of order 3. The group $G$ generated by $a$ and $t$, introduced in [FG85], is called the Fabrykowski-Gupta group. It is known to be a just infinite group, regular branched over $G^{\prime}$ (see [BG02], Proposition 6.2).

Note that $\phi(G)$ is a subgroup of $G$ ₹ ; we still call $\phi$ the decomposition map of $G$

$$
\phi: G \hookrightarrow G \imath A ; \quad g \mapsto\left\langle\left\langle g_{i}\right\rangle\right\rangle_{i \in A} \sigma .
$$

Let $\operatorname{Stab}(n)$ be the subgroup of $G$ that fixes the $n^{\text {th }}$ level $A^{n}$ of $A^{*}$. Then $G=$ $\operatorname{Stab}(1) \rtimes\langle a\rangle$. Furthermore, $\operatorname{Stab}(1)=\langle t\rangle^{\langle a\rangle}$. Let $t_{0}=t=\langle\langle a, 1, t\rangle\rangle, t_{1}=t^{a}=$ $\langle\langle t, a, 1\rangle\rangle$ and $t_{2}=t^{a^{2}}=\langle\langle 1, t, a\rangle\rangle$ be the generators of Stab(1). Clearly $a, t$, $t_{1}, t_{2}$ all have order 3 ; so $G$ is a quotient of $\mathbb{Z} / 3 * \mathbb{Z} / 3$ and $\operatorname{Stab}(1)$ is a quotient of $\mathbb{Z} / 3 * \mathbb{Z} / 3 * \mathbb{Z} / 3$. We call words elements of these free products. Every word $w=w\left(a^{ \pm 1}, t^{ \pm 1}\right)$ uniquely decomposes as

$$
w=t_{c_{1}}^{\gamma_{1}} t_{c_{2}}^{\gamma_{2}} \ldots t_{c_{n}}^{\gamma_{n}} \tau, \quad \text { with } \gamma_{i} \in A^{\times}, c_{i} \in A, c_{i} \neq c_{i+1} \text {, and } \tau \in A,
$$

so that the decomposition map $\phi$ is defined without ambiguity as a map $W \rightarrow W^{3} \times A$ on the set of words.

We define a word metric on $G$ and on $W$ by assigning the following weights on the generators of $G: \ell\left(t^{ \pm 1}\right)=1$ and $\ell\left(a^{ \pm 1}\right)=0$. Then the length of a word $w \in W$, decomposed as in (1), is $\ell(w)=n$. That is, the length of $w$ is the number of letters " $t \pm 1$ " that appear in $w$. The induced metric on $G$ is

$$
\ell(g)=\min \left\{\ell(w) \mid w={ }_{G} g\right\},
$$

for every $\gamma \in G$. We then fix a minimal-length normal form $G \rightarrow W ; g \mapsto w$ on $G$.

Note that $\sum_{i \in A} \ell\left(g_{i}\right) \leq \ell(g)$ for every $g \in G$. We will say that $g \in G$ admits length reduction if there is a $d$ such that

$$
\sum_{i \in A^{d}} \ell\left(g_{i}\right)<\ell(g)
$$

where the $g_{i}$ 's are the states of $g$ on the $d^{\text {th }}$ level of the tree (i.e., the components of $\left.\phi^{d}(g)\right)$. 


\section{Subexponential growth of self-similar groups}

We call a group "self-similar" if it comes equipped with an embedding $\phi: G \rightarrow$ $G$ 々 $\operatorname{Sym}(d)$; this definition covers the Fabrykowski-Gupta example ${ }^{1}$.

A "traditional" way (introduced by Grigorchuk in [Gri84]) to prove that a selfsimilar group $G$ has subexponential growth is to show that every group element admits a fixed proportion of length reduction. More explicitly,

Proposition 4 ([BP06]). Let $G$ be a self-similar group acting on a $d$-regular tree, with a word metric $\ell$. If there exist constants $0 \leq \eta<1$ and $k \geq 0$ such that, for the natural embedding $\phi: \operatorname{Stab}(1) \hookrightarrow G^{d}: g \mapsto\left\langle\left\langle g_{1}, \ldots, g_{d}\right\rangle\right\rangle$,

$$
\sum_{i=1}^{d} \ell\left(g_{i}\right) \leq \eta \ell(g)+k
$$

for every $g \in \operatorname{Stab}(1)$, then $G$ has subexponential growth.

3.1. Length reduction and subexponential growth. Let $G$ be a finitely generated self-similar group acting on a $d$-regular rooted tree, and let $\ell$ be a proper seminorm on $G$. Suppose that for every $g=\left\langle\left\langle g_{1}, \ldots, g_{d}\right\rangle\right\rangle \sigma$ in $G$, we have $\sum_{i=1}^{d} \ell\left(g_{i}\right) \leq \ell(g)$.

Let $\mathcal{I}_{n}$ be the subset of $G$ of elements that have no length reduction up to the $n^{\text {th }}$ level of the tree. It is defined recursively by $\mathcal{I}_{0}=G$ and

$$
\begin{aligned}
\mathcal{I}_{n}=\left\{g=\left\langle\left\langle g_{1}, \ldots, g_{d}\right\rangle\right\rangle \sigma \in G \mid\right. & \sum_{i=1}^{d} \ell\left(g_{i}\right)=\ell(g) \text { and } \\
& \left.g_{i} \in \mathcal{I}_{n-1} \text { for every } 1 \leq i \leq d\right\} .
\end{aligned}
$$

Then $\mathcal{I}_{\infty}:=\bigcap_{n \geq 0} \mathcal{I}_{n}$ is the set of elements that have no length reduction on any level of the tree.

Proposition 5. Let $G=\langle X\rangle$ be a group as above, with $X$ finite, and $X \subset \mathcal{I}_{\infty}$. If there exists some $m$ such that $\mathcal{I}_{m}$ has subexponential growth, then $G$ has subexponential growth.

Moreover, if $\mathcal{I}_{m}$ has linear growth, then the growth of $G$ is bounded in the following way:

$$
\gamma(n) \lesssim e^{n \frac{(\log \log n)^{2}}{\log n}}
$$

where $\gamma(n)=\#\{g \in G \mid \ell(g) \leq n\}$.

Remark. The idea behind this result is the following: if $\mathcal{I}_{m}$ grows subexponentially, then, expressing any group element $g$ of length $n$ as a word in $\mathcal{I}_{m}^{k}$ for some $k$, either $k$ is much smaller than $n$, and thus the set of such words grows slowly; or $k$ is not

\footnotetext{
${ }^{1}$ According to this definition, every group admits a (trivial) self-similarity structure; though that structure will usually not satisfy the additional conditions of Proposition 4 , for example.
} 
negligible compared to $n$ and, in that case, $g$ behaves as in Proposition 4. This kind of argument was used (among other works) in [Bar03]. Anna Erschler has obtained in [Ers04] some similar upper bounds.

In order to prove Proposition 5, we find it useful to state two lemmas.

Lemma 6. Let $F$ be a map such that $\log F$ is concave. Then, for every $n_{1}, \ldots, n_{k}$,

$$
\prod_{i=1}^{k} F\left(n_{i}\right) \leq F\left(\frac{\sum_{i=1}^{k} n_{i}}{k}\right)^{k} .
$$

In particular, if $F$ is subexponential, then there is a subexponential map $G \geq F$ such that $\log G$ is concave, and hence $G$ satisfies Equation (2).

Proof. By hypothesis, $\sum_{i=1}^{k} \log F\left(n_{i}\right) \leq k \log F\left(\frac{\sum_{i=1}^{k} n_{i}}{k}\right)$. Exponentiating this last equation, the desired inequality follows.

Suppose now that $F$ is subexponential, that is, $\lim _{n \rightarrow \infty} \frac{\log F(n)}{n}=0$. Let $\left(\varepsilon_{i}\right)_{i \geq 1}$ be strictly decreasing to zero and $\left(n_{i}\right)_{i \geq 1}$ be strictly increasing, such that $n_{1}=1$ and $\frac{\log F(n)}{n} \leq \varepsilon_{i}$ for every $n \geq n_{i}$. Define then $\log G(n)=\varepsilon_{i} n+\delta_{i}$ on the interval $n_{i} \leq n \leq n_{i+1}$, with $\delta_{1}=0$ and $\delta_{i}=\left(\varepsilon_{i-1}-\varepsilon_{i}\right) n_{i}+\delta_{i-1}$. Then $\log G \geq \log F$ is continuous and concave by definition and $\lim _{n \rightarrow \infty} \frac{\log G(n)}{n}=0$.

Lemma 7. Consider the maps

$$
\lambda(n)=\frac{n \log \log n}{\log n}
$$

and for some $d, m>0$,

$$
\begin{aligned}
f(n)= & \frac{\log n}{n(\log \log n)^{2}}+\frac{d^{m}(\log n)^{2}}{n(\log \log n)^{2}} \\
& +\frac{n-\lambda(n)}{n} \frac{\log n}{\log \left(\frac{n-\lambda(n)}{d^{m}}\right)}\left(\frac{\log \log \left(\frac{n-\lambda(n)}{d^{m}}\right)}{\log \log n}\right)^{2} .
\end{aligned}
$$

Then, there exists an integer $N$ such that $f(n) \leq 1$ for every $n \geq N$.

Proof. We write

$$
f(n)=\frac{1}{\log \log (n)^{2}} \cdot \frac{\log n}{n}(1+A \log n)+\frac{\log \log \left(n^{\prime}\right)^{2}}{\log \log (n)^{2}} \cdot A \frac{n^{\prime} \log n}{n \log \left(n^{\prime}\right)},
$$

with $A=d^{m}$ and $n^{\prime}=(n-\lambda(n)) / A$. Since $\frac{1}{\log \log (n)^{2}}<1$ and $\frac{\log \log \left(n^{\prime}\right)^{2}}{\log \log (n)^{2}}<1$ for $n$ 
large enough, it suffices to prove the stronger inequality

$$
\frac{\log n}{n}(1+A \log n)+A \frac{n^{\prime} \log n}{n \log n^{\prime}}<1
$$

for all $n$ large enough.

Now this amounts to

$$
\frac{\log n}{n}(1+A \log n)<1-\frac{\log n}{\log n^{\prime}}+\frac{\log \log n}{\log n^{\prime}}
$$

if we multiply this last inequality by $\frac{\log n^{\prime}}{\log \log n}$, we get

$$
\frac{\log n \log n^{\prime}}{n \log \log n}(1+A \log n)<1-\frac{\log \left(n / n^{\prime}\right)}{\log \log n} .
$$

Then the left-hand side is bounded above by $(A+1) \frac{\log (n)^{3}}{n \log \log n}$, which tends to 0 as $n \rightarrow \infty$; and $\frac{\log \left(n / n^{\prime}\right)}{\log \log n}$ also tends to 0 as $n \rightarrow \infty$ because $n / n^{\prime}$ tends to $A$, so the right-hand side tends to 1 . It follows that (3) holds for $n$ large enough.

Proof of Proposition 5. We first suppose that $\mathcal{I}_{m}$ has subexponential growth. Let us write every $g \in G$ as a product $g=g_{1} \ldots g_{N(g)}$ with $g_{i} \in \mathcal{I}_{m}$ and where $N(g)=\min \left\{k \mid g=g_{1} \ldots g_{k}, g_{i} \in \mathcal{I}_{m}\right\}$.

For any $\lambda \leq \frac{n}{2}$, the sphere of radius $n$ in $G$ is the union of $W_{\lambda}^{<}(n):=\{g \mid \ell(g)=n, N(g) \leq \lambda\}$ and $W_{\lambda}^{>}(n):=\{g \mid \ell(g)=n, N(g)>\lambda\}$.

Let $\mathcal{I}_{m}\left(n_{i}\right)$ be the sphere of radius $n_{i}$ in $\mathcal{I}_{m}$ and $\delta\left(n_{i}\right)=\# \mathcal{I}_{m}\left(n_{i}\right)$. Then the cardinality of $\mathcal{I}_{m}^{k} \cap\{g \in G \mid \ell(g)=n\}$ is at most $\sum_{n_{1}+\cdots+n_{k}=n} \prod_{i=1}^{k} \delta\left(n_{i}\right)$ for any $k \leq \lambda$. Hence,

$$
\# W_{\lambda}^{<}(n) \leq \sum_{k=1}^{\lambda} \sum_{n_{1}+\cdots+n_{k}=n} \prod_{i=1}^{k} \delta\left(n_{i}\right) .
$$

We may replace $\delta(n)$ by a possibly larger function, still written $\delta(n)$, which is increasing and, by Lemma 6, satisfies Equation (2). Hence,

$$
\begin{aligned}
& \# W_{\lambda}^{<}(n) \leq \sum_{k=1}^{\lambda} \sum_{n_{1}+\cdots+n_{k}=n} \delta\left(\frac{n}{k}\right)^{k}
\end{aligned}
$$

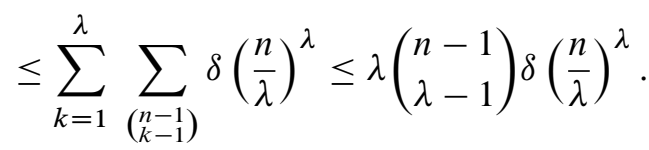


As $\left(\begin{array}{l}n \\ \lambda\end{array}\right) \leq\left(\frac{e n}{\lambda}\right)^{\lambda}$ by Stirling's formula, it follows that

$$
\# W_{\lambda}^{<}(n) \leq e^{\lambda}\left(\frac{n}{\lambda}\right)^{\lambda-1} \delta\left(\frac{n}{\lambda}\right)^{\lambda}
$$

On the other hand,

$$
\# W_{\lambda}^{>}(n) \leq[G: \operatorname{Stab}(m)] \sum_{n_{1}+\cdots+n_{d^{m}} \leq n-\lambda} \gamma\left(n_{1}\right) \ldots \gamma\left(n_{d^{m}}\right) .
$$

If $\gamma=\lim _{n \rightarrow \infty} \gamma(n)^{1 / n}$ is the growth rate of $G$, then there is a constant $K>0$ such that $K \gamma \geq \gamma(n)^{1 / n}$ for every $n \geq 1$. Hence,

$$
\# W_{\lambda}^{>}(n) \leq[G: \operatorname{Stab}(m)] \sum_{n_{1}+\cdots+n_{d^{m}} \leq n-\lambda} K^{d^{m}} \gamma^{n-\lambda}
$$

and

$$
\# W_{\lambda}^{>}(n) \leq p(n) \gamma^{n-\lambda}
$$

where $p(n)=[G: \operatorname{Stab}(m)] K^{d^{m}}\left(\begin{array}{c}n-\lambda \\ d^{m}\end{array}\right)$ is a polynomial (of degree $d^{m}$ ).

Set $\varepsilon=\frac{\lambda}{n}$. From Equations (4) and (5) we get

$$
\begin{aligned}
\gamma & \leq \lim _{n \rightarrow \infty}\left(\# W_{\lambda}^{>}(n)+\# W_{\lambda}^{<}(n)\right)^{1 / n} \\
& \leq \max \left\{\lim _{n \rightarrow \infty} \# W_{\lambda}^{>}(n)^{1 / n}, \lim _{n \rightarrow \infty} \# W_{\lambda}^{<}(n)^{1 / n}\right\} \leq \max \left\{\varepsilon^{-\varepsilon} \delta\left(\varepsilon^{-1}\right)^{\varepsilon}, \gamma^{1-\varepsilon}\right\} .
\end{aligned}
$$

As $\lim _{\varepsilon \rightarrow 0} \varepsilon^{-\varepsilon} \delta\left(\varepsilon^{-1}\right)^{\varepsilon}=1$, we obtain in all cases $\gamma=1$.

Suppose next that $\mathcal{I}_{m}$ grows linearly. We have to show that there exist constants $A, B>0$ such that

$$
\gamma(n) \leq \exp \left(A+B \frac{n(\log \log n)^{2}}{\log n}\right)
$$

for $n$ large enough.

Consider the subexponential map $F(n)=e^{\frac{n(\log \log n)^{2}}{\log n}}$. Then, for $n \geq c:=e^{e^{2}}$,

$$
\begin{gathered}
(\log F(n))^{\prime \prime}=\frac{1}{n(\log n)^{3}}\left(-\log n(\log \log n)^{2}+2 \log n \log \log n\right. \\
\left.+2(\log \log n)^{2}-6 \log \log n+2\right) \leq 0
\end{gathered}
$$

so that $\log F(n)$ is concave for $n \geq c$. 
Define $A=\log \gamma(N)$, where $N$ is as in Lemma 7. Consider also the constants

and

$$
M=\left(d^{m}+1\right)[G: \operatorname{Stab}(m)] \gamma(c)^{d^{m}}\left(\frac{e}{d^{m}}\right)^{d^{m}}
$$

$$
B=\max \left\{2+\log \delta\left(\frac{n}{\lambda}\right), \log M+\left(d^{m}-1\right) A+\log 2\right\} .
$$

Define then the map

$$
F(n)= \begin{cases}\exp \left(A+B \frac{n(\log \log n)^{2}}{\log n}\right) & \text { if } n \geq c, \\ \exp \left(A+B \frac{c(\log \log c)^{2}}{\log c}\right) & \text { if } 0 \leq n<c,\end{cases}
$$

so that $\gamma(k) \leq F(k)$ for every $k \leq N$. For $n>N$, let us show by induction that $\gamma(n) \leq F(n)$.

As before, we have

$$
\begin{aligned}
\# W_{\lambda}^{>}(n) & \leq[G: \operatorname{Stab}(m)] \sum_{n_{1}+\cdots+n_{d^{m}} \leq n-\lambda} \gamma\left(n_{1}\right) \ldots \gamma\left(n_{d^{m}}\right) \\
& \leq[G: \operatorname{Stab}(m)] \sum_{n_{1}+\cdots+n_{d^{m}} \leq n-\lambda} F\left(n_{1}\right) \ldots F\left(n_{d^{m}}\right) .
\end{aligned}
$$

Developing this last sum and thanks to Lemma 6, we get

$$
\# W_{\lambda}^{>}(n) \leq[G: \operatorname{Stab}(m)]\left(\begin{array}{c}
n-\lambda \\
d^{m}
\end{array}\right)\left(F(c)^{d^{m}}+\sum_{k=1}^{d^{m}} F(c)^{d^{m}-k} F\left(\frac{n-\lambda}{k}\right)^{k}\right) .
$$

Hence,

$$
\# W_{\lambda}^{>}(n) \leq\left(d^{m}+1\right)[G: \operatorname{Stab}(m)] F(c)^{d^{m}}\left(\begin{array}{c}
n-\lambda \\
d^{m}
\end{array}\right) F\left(\frac{n-\lambda}{d^{m}}\right)^{d^{m}} .
$$

Thus, as $\left(\begin{array}{c}n-\lambda \\ d^{m}\end{array}\right) \leq\left(\frac{e(n-\lambda)}{d^{m}}\right)^{d^{m}}<\left(\frac{e}{d^{m}}\right)^{d^{m}} n^{d^{m}}$, we get

$$
\# W_{\lambda}^{>}(n) \leq M n^{d^{m}} F\left(\frac{n-\lambda}{d^{m}}\right)^{d^{m}}
$$

Together with (4), this gives

$$
\gamma(n) \leq \# W_{\lambda}^{<}(n)+\# W_{\lambda}^{>}(n) \leq\left(\frac{n}{\lambda}\right)^{\lambda-1} \delta\left(\frac{n}{\lambda}\right)^{\lambda}+M n^{d^{m}} F\left(\frac{n-\lambda}{d^{m}}\right)^{d^{m}}
$$

For $\lambda=\frac{n \log \log n}{\log n}$, we see that $(\lambda-1) \log \left(\frac{n}{\lambda}\right)+\lambda \log \delta\left(\frac{n}{\lambda}\right) \leq A-\log 2+B \frac{n(\log \log n)^{2}}{\log n}$, and hence

$$
\left(\frac{n}{\lambda}\right)^{\lambda-1} \delta\left(\frac{n}{\lambda}\right)^{\lambda} \leq \frac{1}{2} F(n) .
$$


It remains to verify that

$$
M n^{d^{m}} F\left(\frac{n-\lambda}{d^{m}}\right)^{d^{m}} \leq \frac{1}{2} F(n)
$$

but this is equivalent to

$$
\begin{gathered}
\frac{\left(\log M+\left(d^{m}-1\right) A+\log 2\right) \log n}{B n(\log \log n)^{2}}+\frac{d^{m}(\log n)^{2}}{B n(\log \log n)^{2}} \\
+\frac{n-\lambda}{n} \frac{\log n}{\log \left(\frac{n-\lambda}{d^{m}}\right)}\left(\frac{\log \log \left(\frac{n-\lambda}{d^{m}}\right)}{\log \log n}\right)^{2} \leq 1
\end{gathered}
$$

As the left side of (6) is smaller than $f(n)$ by definition of $B$, this holds by Lemma 7 .

\section{Growth of the Fabrykowski-Gupta group}

In the remainder, $G$ will denote the Fabrykowski-Gupta group, as defined in Section 2. We first reduce Theorem 1 to a statement on "incompressible" words $\mathcal{I}_{\infty}$.

Proof of Theorem 1. The lower bound is easily computed. Indeed, consider the morphism $\psi: G^{\prime} \rightarrow G^{\prime}$ induced by $a \mapsto t$ and $t \mapsto t^{a}$, where $G^{\prime}=\left\langle t^{ \pm a^{i}} t^{\mp a^{j}}, i \neq j\right\rangle$. Since $\psi\left(t^{ \pm a^{i}} t^{\mp a^{j}}\right)=\left\langle\left\langle t^{ \pm a^{i}} t^{\mp a^{j}}, 1,1\right\rangle\right\rangle$, there is an injective map

$$
\left(B_{G}(n) \cap G^{\prime}\right)^{3} \hookrightarrow B_{G}(6 n) \cap G^{\prime} ; \quad\left(g_{1}, g_{2}, g_{3}\right) \mapsto \psi\left(g_{1}\right) \psi\left(g_{2}\right)^{a} \psi\left(g_{3}\right)^{a^{2}}
$$

where $B_{G}(n)$ is the ball of radius $n$ in $G$. Hence, $\beta(6 n) \geq \beta(n)^{3}$, with $\beta(n)=$ $\#\left(B_{G}(n) \cap G^{\prime}\right)$. Iterating this inequality, one get $\beta\left(2 \cdot 6^{n}\right) \geq \beta(2)^{3^{n}}=12^{3^{n}}$, so that

$$
\gamma(t) \geq \beta(t) \geq 12^{(t / 2)^{\frac{\log 3}{\log 6}} .} .
$$

On the other hand, the upper bound follows directly from the following result and Proposition 5.

Proposition 8. (1) If $w \notin \mathcal{I}_{\infty}$, then $w$ has length reduction up to the third level. Equivalently, $I_{\infty}=I_{3}$.

(2) The growth of $\mathcal{I}_{\infty}$ is linear.

Before we prove Proposition 8, let us give some definitions and lemmas. It will also be more convenient to work with reduced words $R$ than with group elements; by definition, a reduced word is a word over the alphabet $A^{\times} \cup\left\{t, t^{-1}\right\}$, with no two 
consecutive elements of $A$; reduction amounts to replacing two consecutive elements of $A$ by their product, and deleting $1 \in A$.

With this definition, $\ell(w)$ counts the number of $t^{ \pm 1}$ in $w$; there is again a decomposition map $\phi: R \rightarrow R^{A} \times \operatorname{Sym}(A)$, written $w \mapsto\left\langle\left\langle w_{0}, w_{1}, w_{2}\right\rangle\right\rangle \sigma$; and

$$
\mathcal{I}_{1}=\left\{w \in R \mid \text { there are no consecutive } t^{ \pm 1} \text { in the } w_{i}{ }^{\prime} \mathrm{s}\right\} .
$$

4.1. Length reduction of words. Consider the subsets of $A^{*}$

$$
S=\left\{s \mid s \text { is a subword of }(\ldots 21021012012 \ldots)^{\sigma} \text {, for some } \sigma \in A\right\}
$$

and

$$
\partial S=\{s \mid s \text { is a subword of }(\ldots 111222 \ldots)\}
$$

Note that

$$
s=\left(s_{i}\right)_{i=1}^{n} \in A \partial S \quad \text { if and only if } \quad \Sigma s:=\left(-\sum_{k=1}^{i} s_{k}\right)_{i=1}^{n} \in \mathcal{S} .
$$

For sequences $c=\left(c_{i}\right)_{i=1}^{n} \in S$ and $\gamma=\left(\gamma_{i}\right)_{i=1}^{n} \in \partial S$, consider the maps

$$
m(c)= \begin{cases}1 & \text { if } c \text { is a subword of }(012)^{\infty}, \\ k & \text { if } c_{k-1}=c_{k+1}, \\ n & \text { if } c \text { is a subword of }(021)^{\infty},\end{cases}
$$

and

$$
\partial m(\gamma)= \begin{cases}1 & \text { if } \gamma \text { is a subword of } 2^{\infty} \\ k & \text { if } \gamma_{k}=1 \text { and } \gamma_{k+1}=2 \\ n & \text { if } \gamma \text { is a subword of } 1^{\infty}\end{cases}
$$

so that, obviously,

$$
\partial m(\gamma)=m(\Sigma \gamma)
$$

Consider a word $w$ over $A^{\times} \cup\left\{t, t^{-1}\right\}$. Up to reduction, it may uniquely be written as

$$
w=t_{c_{1}}^{\gamma_{1}} \ldots t_{c_{n}}^{\gamma_{n}} \tau \quad \text { with } c_{i} \in A, \gamma_{i} \in\{ \pm 1\}, \text { and } \tau \in A,
$$

where $t_{i}=a^{-i} t a^{i}$. Define the exponent sequence of $w$ as $\gamma(w)=\left(\gamma_{i}\right)_{i=1}^{n}$, and the index sequence of $w$ as $c(w)=\left(c_{i}\right)_{i=1}^{n}$.

As $t_{\sigma(i)}=t_{i}^{\sigma}$ for any $i, \sigma \in A$, the following remark holds.

Lemma 9. Let $s=t_{c_{1}}^{\gamma_{1}} \ldots t_{c_{n}}^{\gamma_{n}} \tau=\left\langle\left\langle s_{0}, s_{1}, s_{2}\right\rangle \tau \tau\right.$ be any word and its first level decomposition, and let $\sigma \in A$. Then

$$
s_{\sigma}:=t_{\sigma\left(c_{1}\right)}^{\gamma_{1}} \ldots t_{\sigma\left(c_{n}\right)}^{\gamma_{n}} \tau=\left\langle\left\langle s_{0}, s_{1}, s_{2}\right\rangle\right\rangle^{\sigma} \tau .
$$

In particular, $s$ and $s_{\sigma}$ have the same first level decompositions up to a permutation of the components. 
We may now characterize $\mathcal{I}_{1}$ in the following way.

Lemma 10. The set $\mathcal{I}_{1}$ is exactly the set of words (9) such that

(a) $c(w) \in \mathcal{S}$;

(b) denoting $m=m(c(w))$, if $2<m<n-1$, then $\gamma(w)_{m-1}=\gamma(w)_{m+1}$.

Proof. Suppose first that $w$ satisfies (a). Then all $t$ 's in the $w_{j}$ are separated by precisely one element in $A^{\times}$, so that two $t^{ \pm 1}$ 's are adjacent; except possibly for the $t$ coming from $t_{c_{m}}^{\gamma_{m}}$. Locally, we see there

$$
\begin{aligned}
w & =\ldots t_{\sigma(1)}^{\gamma_{m-1}} t_{\sigma(0)}^{\gamma_{m}} t_{\sigma(1)}^{\gamma_{m+1}} \ldots \\
& =\ldots\left\langle\left\langle t^{\gamma_{m-1}} a^{\gamma_{m}} t^{\gamma_{m+1}}, a^{\gamma_{m-1}+\gamma_{m+1}}, t^{\gamma_{m}}\right\rangle\right\rangle^{\sigma} \ldots,
\end{aligned}
$$

and since $\gamma_{m-1}+\gamma_{m+1} \in A^{\times}$by (b) there are no adjacent $t^{ \pm 1}$ 's there either.

Conversely, suppose that $w$ does not satisfy (a). Then either $c_{i}=c_{i+1}$ for some $i$, or $w$ contains a subword

$$
\begin{aligned}
u & =t_{\sigma(0)}^{\alpha} t_{\sigma(1)}^{\beta} t_{\sigma(0)}^{\gamma} \\
& =\left\langle\left\langle a^{\alpha}, 1, t^{\alpha}\right\rangle\right\rangle^{\sigma}\left\langle\left\langle t^{\beta}, a^{\beta}, 1\right\rangle\right\rangle^{\sigma}\left\langle\left\langle a^{\gamma}, 1, t^{\gamma}\right\rangle\right\rangle^{\sigma} \\
& =\left\langle\left\langle a^{\alpha} t^{\beta} a^{\gamma}, a^{\beta}, t^{\alpha} t^{\gamma}\right\rangle\right\rangle^{\sigma},
\end{aligned}
$$

and in both cases there are neighbouring $t^{ \pm 1}$ 's in some $u_{j}$.

Finally, suppose that $w$ satisfies (a) but not (b); then $2<m<n-1$ and $w$ contains a subword

$$
v=t_{\sigma(2)}^{\alpha} t_{\sigma(1)}^{\beta} t_{\sigma(0)}^{\gamma} t_{\sigma(1)}^{-\beta} t_{\sigma(2)}^{\delta}=\left\langle\left\langle t^{\beta} a^{\gamma} t^{-\beta}, t^{\alpha} t^{\delta}, a^{\alpha} t^{\gamma} a^{\delta}\right\rangle\right\rangle^{\sigma},
$$

and again there are neighbouring $t^{ \pm 1}$, s in some $v_{j}$.

Consider now a word $w \in S$ as in (9), with $m(c(g))=m$ and $2<m<n-1$. Developing $w$ on the first level, we get

$$
\begin{aligned}
& \left\langle\left\langle w_{0}, w_{1}, w_{2}\right\rangle\right\rangle \\
& \left.=\ldots\left\langle 1, t^{\gamma_{m-2}}, a^{\gamma_{m-2}}\right\rangle\right\rangle^{a^{c m}}\left\langle\left\langle t^{\gamma_{m-1}}, a^{\gamma_{m-1}}, 1\right\rangle\right\rangle^{c^{c m}} \\
& \quad\left\langle\left\langle a^{\gamma_{m}}, 1, t^{\gamma_{m}}\right\rangle\right\rangle^{a^{c_{m}}}\left\langle\left\langle t^{\gamma_{m+1}}, a^{\gamma_{m+1}}, 1\right\rangle\right\rangle^{a^{c m}}\left\langle\left\langle 1, t^{\gamma_{m+2}}, a^{\gamma_{m+2}}\right\rangle\right\rangle^{a^{c_{m}}} \ldots
\end{aligned}
$$

By Lemma 9, up to a permutation of the components, we may suppose $c_{m}=0$. We may also suppose that $c_{1}=2$, as the other two cases behave similarly, and we will 
do so for the remainder of the section. Hence we get

$$
\begin{aligned}
w_{0} & =t^{\gamma_{2}} a^{\gamma_{3}} t^{\gamma_{5}} a^{\gamma_{6}} \ldots a^{\gamma_{m-3}} t^{\gamma_{m-1}} a^{\gamma_{m}} t^{\gamma_{m+1}} a^{\gamma_{m+3}} t^{\gamma_{m+4}} \ldots \\
& =t_{0}^{\gamma_{2}} t_{-\gamma_{3}}^{\gamma_{5}} \ldots t_{*}^{\gamma_{m-4}} t_{*-\gamma_{m-3}}^{\gamma_{m-1}} t_{*-\gamma_{m-3}-\gamma_{m}}^{\gamma_{m}+t_{*-\gamma_{m}-3}-\gamma_{m}-\gamma_{m+3}} \ldots \\
w_{1} & =t^{\gamma_{1}} a^{\gamma_{2}} t^{\gamma_{4}} a^{\gamma_{5}} \ldots a^{\gamma_{m-4}} t^{\gamma_{m-2}} a^{\gamma_{m-1}+\gamma_{m+1}} t^{\gamma_{m}+2} a^{\gamma_{m}+4} t^{\gamma_{m+5}} \ldots \\
& =t_{0}^{\gamma_{1}} t_{-\gamma_{2}}^{\gamma_{4}} \ldots t_{*}^{\gamma_{m-5}} t_{*-\gamma_{m-4}}^{\gamma_{m-2}} t_{*-\gamma_{m-4}-\left(\gamma_{m-1}+\gamma_{m+1}\right)}^{\gamma_{m-2}} t_{*-\gamma_{m-4}-\left(\gamma_{m-1}+\gamma_{m+1}\right)-\gamma_{m+4}}^{\gamma_{m}} \ldots \\
w_{2} & =a^{\gamma_{1}} t^{\gamma_{3}} a^{\gamma_{4}} t^{\gamma_{6}} \ldots a^{\gamma_{m-5}} t^{\gamma_{m-3}} a^{\gamma_{m-2}} t^{\gamma_{m}} a^{\gamma_{m+2}} t^{\gamma_{m+3}} \ldots \\
& =t_{-\gamma_{1}}^{\gamma_{3}} t_{-\gamma_{1}-\gamma_{4}}^{\gamma_{6}} \ldots t_{*}^{\gamma_{m-3}} t_{*-\gamma_{m-2}}^{\gamma_{m}} t_{*-\gamma_{m-2}-\gamma_{m+2}}^{\gamma_{m+3}} t_{*-\gamma_{m-2}-\gamma_{m+2}-\gamma_{m+5}}^{\gamma_{m}} \ldots
\end{aligned}
$$

Note that our assumptions $c_{m}=0, c_{1}=2$ imply $m \equiv 0(\bmod 3)$. We also have $\ell\left(w_{i}\right)=\lfloor(n+2-i) / 3\rfloor$. We get

$$
\begin{aligned}
& \gamma\left(w_{0}\right)=\left(\gamma_{2} \gamma_{5} \ldots \gamma_{m-4} \gamma_{m-1} \gamma_{m+1} \gamma_{m+4} \ldots\right), \\
& \gamma\left(w_{1}\right)=\left(\gamma_{1} \gamma_{4} \ldots \gamma_{m-5} \gamma_{m-2} \gamma_{m+2} \gamma_{m+5} \ldots\right) \text {, } \\
& \gamma\left(w_{2}\right)=\left(\gamma_{3} \gamma_{6} \ldots \gamma_{m-3} \gamma_{m} \gamma_{m+3} \gamma_{m+6} \ldots\right) \text {, }
\end{aligned}
$$

and, writing $\tilde{\gamma}\left(w_{0}\right)=\left(\gamma_{2} \ldots \gamma_{m-4}\left(\gamma_{m-1}+\gamma_{m+1}\right) \gamma_{m+4} \ldots\right)$,

$$
\begin{aligned}
& c\left(w_{0}\right)=\Sigma\left(0 \gamma_{3} \ldots \gamma_{m-3} \gamma_{m} \gamma_{m+3} \ldots\right), \text { a prefix of } \Sigma\left(0 \gamma\left(w_{2}\right)\right), \\
& c\left(w_{1}\right)=\Sigma\left(0 \gamma_{2} \ldots \gamma_{m-4}\left(\gamma_{m-1}+\gamma_{m+1}\right) \gamma_{m+4} \ldots\right), \text { a prefix of } \Sigma\left(0 \tilde{\gamma}\left(w_{0}\right)\right), \\
& c\left(w_{2}\right)=\Sigma\left(\gamma_{1} \gamma_{4} \ldots \gamma_{m-2} \gamma_{m+2} \gamma_{m+5} \ldots\right), \text { a prefix of } \Sigma\left(\gamma\left(w_{1}\right)\right) .
\end{aligned}
$$

If $\tilde{\gamma}\left(w_{0}\right), \gamma\left(w_{1}\right), \gamma\left(w_{2}\right) \in A \partial S$, then $c\left(w_{0}\right), c\left(w_{1}\right), c\left(w_{2}\right) \in S$, and conversely. Furthermore, in this case, the following hold by (8):

$$
\begin{aligned}
& m_{0}:=m\left(c\left(w_{0}\right)\right)=\partial m\left(\gamma\left(w_{2}\right)\right)+1, \\
& m_{1}:=m\left(c\left(w_{1}\right)\right)=\partial m\left(\tilde{\gamma}\left(w_{0}\right)\right)+1, \\
& m_{2}:=m\left(c\left(w_{2}\right)\right)=\partial m\left(\gamma\left(w_{1}\right)\right) .
\end{aligned}
$$

Lemma 11. The following are equivalent:

(1) $w \in \mathcal{I}_{\infty}$;

(2) $c\left(w_{x}\right) \in S$ for all $x \in A^{*}$;

(3) $\gamma\left(w_{x i}\right) \in A \partial S$ and $\tilde{\gamma}\left(w_{x 0}\right) \in A \partial S$ for all $x \in A^{*}, i \in A^{\times}$.

Proof. (1) $\Longrightarrow$ (2) follows from Lemma 10, because $w \in \mathcal{I}_{\infty}$ implies $w_{x} \in \mathcal{I}_{\infty}$ for all $x \in A^{*}$.

For $(2) \Longrightarrow(1)$, we show that $w_{x} \in \mathcal{I}_{1}$ for all $x \in A^{*}$; in view of Lemma 10, it suffices to show that if $w_{x}=t_{c_{1}}^{\gamma_{1}} \ldots t_{c_{n}}^{\gamma_{n}} \tau$, and $m\left(c\left(w_{x}\right)\right)=m \in\{3, \ldots, n-2\}$, then $\gamma_{m-1}=\gamma_{m+1}$. But if not, we would have $c\left(w_{x i}\right)=\ldots j j \ldots$ for some $i \in A$, so $c\left(w_{x i}\right) \notin S$.

The equivalence (2) $\Longleftrightarrow$ (3) follows from comparing (10) and (11). 
Lemma 12. Let $w \in \mathcal{I}_{\infty}$ be such that $\gamma(w) \in A \partial S$, say $\partial m(\gamma(w))=p$. Then $p \leq 11$ or $p \geq n-10$.

Proof. As above, we assume that $c_{m}=0$ and $c_{1}=2$, and derive from these assumptions that $p \leq 9$ or $p \geq n-8$; the general case follows by adding up to two symbols $t_{*}$ at the head or tail of $w$.

Assume for contradiction that $9<p<n-8$. From the assumption $w \in \mathcal{I}_{\infty}$, we know that $c\left(w_{i}\right) \in S$ for all $i \in A$; so $m_{0}, m_{1}, m_{2}$ are defined. Now that means that both sequences $\tilde{\gamma}\left(w_{0}\right)$ and $\gamma\left(w_{0}\right)$ belong to $A \partial S$, the latter because it is a subsequence of $\gamma(w)$. Therefore, $m-4 \leq p \leq m+3$; and furthermore $p \notin\{m-1, m\}$ because $\gamma_{m-1} \neq \gamma_{m+1}$. We may then explicitly compute:

\begin{tabular}{c|ccc}
$p$ & $m_{0}$ & $m_{1}$ & $m_{2}$ \\
\hline$m-4$ & $\frac{m}{3}-1$ & $\frac{m}{3}+1$ & $\frac{m}{3}-1$ \\
$m-3$ & $\frac{m}{3}$ & $\frac{m}{3}+1$ & $\frac{m}{3}-1$ \\
$m-2$ & $\frac{m}{3}$ & $\frac{m}{3}+1$ & $\frac{m}{3}$ \\
$m+1$ & $\frac{m}{3}+1$ & $\frac{m}{3}$ & $\frac{m}{3}$ \\
$m+2$ & $\frac{m}{3}+1$ & $\frac{m}{3}$ & $\frac{m}{3}+1$ \\
$m+3$ & $\frac{m}{3}+2$ & $\frac{m}{3}$ & $\frac{m}{3}+1$.
\end{tabular}

By our assumptions, $2<m_{0}<n-1$; but we may check, in each of the cases, that $\gamma\left(w_{0}\right)_{m_{0}-1} \neq \gamma\left(w_{0}\right)_{m_{0}+1}$. By Lemma 10, this implies that $w_{0} \notin \mathcal{I}_{1}$; so $w \notin \mathcal{I}_{\infty}$.

Proof of Proposition 8. We first consider $w=t_{c_{1}}^{\gamma_{1}} \ldots t_{c_{m}}^{\gamma_{m}} \ldots t_{c_{n}}^{\gamma_{n}} \tau \in \mathcal{I}_{3}$, with $m(c(w))=m$. For every $i \in A$ and $j \in A^{\times}$, we know by Lemma 11 that $\gamma\left(w_{i}\right)$, $\gamma\left(w_{i j}\right), \tilde{\gamma}\left(w_{0}\right), \tilde{\gamma}\left(w_{i 0}\right) \in A \partial S$.

Again by Lemma 11, all we have to show is that $\gamma\left(w_{x j}\right) \in A \partial S$ and $\tilde{\gamma}\left(w_{x 0}\right) \in A \partial S$ for all $j \in A^{\times}$and $x \in A^{*}$ of length at least 2. Now, for $j \in A^{\times}$, as $\gamma\left(w_{0 j}\right) \in A \partial S$, the index sequence $\gamma\left(w_{0}\right)$ is of one of the following types:

$\begin{array}{cccccccc}\ldots & \gamma_{m-7} & \gamma_{m-4} & \gamma_{m-1} & \gamma_{m+1} & \gamma_{m+4} & \gamma_{m+7} & \ldots \\ \ldots & 1 & 2 & 1 & 1 & 2 & 2 & \ldots \\ \ldots & 1 & 1 & 1 & 1 & 2 & 2 & \ldots \\ \ldots & 1 & 1 & 2 & 2 & 2 & 2 & \ldots \\ \ldots & 1 & 1 & 2 & 2 & 1 & 2 & \ldots\end{array}$

which means that

$$
\partial m\left(\tilde{\gamma}\left(w_{0}\right)\right) \in\left\{\frac{m}{3}-2, \frac{m}{3}-1, \frac{m}{3}, \frac{m}{3}+1\right\} .
$$

In any of those cases, note that we also have $\gamma\left(w_{00}\right) \in A \partial S$. Altogether, this implies that $\gamma\left(w_{0 y}\right) \in A \partial S$ for every $y \in A^{*}$. Hence, $\gamma\left(w_{0 x i}\right) \in A \partial S$ for $i \in A^{\times}$and $\tilde{\gamma}\left(w_{0 \times 0}\right) \in A \partial S$ for every $x \in A^{*}$. 
Next, for $i \in A^{\times}$, since $\gamma\left(w_{i}\right) \in A \partial S$, we have $\gamma\left(w_{i y}\right) \in A \partial S$ for every $y \in A^{*}$. Hence, $\gamma\left(w_{i x j}\right) \in A \partial S$ for $j \in A^{\times}$and $\tilde{\gamma}\left(w_{i x 0}\right) \in A \partial S$ for every $x \in A^{*}$.

Moreover, $\gamma\left(w_{i j}\right) \in A \partial S$ for all $i \in A^{\times}$and $j \in A$ imply that

$$
\begin{aligned}
& m\left(c\left(w_{1}\right)\right)-\partial m\left(\gamma\left(w_{1}\right)\right) \in\{-3,-2,-1,2,3,4\}, \\
& m\left(c\left(w_{2}\right)\right)-\partial m\left(\gamma\left(w_{2}\right)\right) \in\{-3,-2,-1,2,3,4\} .
\end{aligned}
$$

Using relations (12), (13), (14) and (15), we see that, given one of $m(c(w))$, $m\left(c\left(w_{0}\right)\right), m\left(c\left(w_{1}\right)\right)$ or $m\left(c\left(w_{2}\right)\right)$, the number of possibilities of choosing the three others (so that $w$ remains in $\mathcal{I}_{\infty}$ ) is bounded by a constant $K$, independent of the length of $w$.

We are now ready to show that $\delta(n)=\# \mathcal{I}_{\infty}(n)$ is bounded by a constant, independent of $n$. Indeed consider $w \in \mathcal{I}_{\infty}(n)$. There are a priori $n$ possibilities for $c(w)$, according to Lemma 10. Furthermore, for $i \in A^{\times}$, we know by Lemma 11 that $\gamma\left(w_{i}\right) \in A \partial S$. Hence, by Lemma 12, we have $m\left(c\left(w_{i}\right)\right) \leq 11$ or $m\left(c\left(w_{i}\right)\right) \geq n-10$. Therefore, there is at most 20 choices for $m\left(c\left(w_{i}\right)\right)$ (to be chosen between 1 and $n$ ). Now, by the previous paragraph, there are at most $20 L$ choices for all other $c\left(w_{i}\right)$, and in particular for $c(w)$.

There are also only boundedly many possibilities (say $K$ ) for $\gamma\left(w_{i}\right)$; indeed $\gamma\left(w_{1}\right), \gamma\left(w_{2}\right)$ and $\tilde{\gamma}\left(w_{0}\right)$ are determined by the $c\left(w_{i}\right)$, and there are at most six possibilities for $\gamma\left(w_{i}\right)$ once $\tilde{\gamma}\left(w_{0}\right)$ is known.

All in all, we have obtained $\delta(n) \leq 6 \cdot 20 K$, independently of $n$.

\section{References}

[Bar03] L. Bartholdi, A Wilson group of non-uniformly exponential growth. C. R. Math. Acad. Sci. Paris 336 (2003), 549-554. Zbl 1050.20018 MR 1981466

[BG02] L. Bartholdi and R. I. Grigorchuk, On parabolic subgroups and Hecke algebras of some fractal groups. Serdica Math. J. 28 (2002), 47-90. Zbl 1011.20028 MR 1899368

[BP06] K.-U. Bux and R. Pérez, On the growth of iterated monodromy groups. In Topological and asymptotic aspects of group theory, Contemp. Math. 394, Amer. Math. Soc., Providence, RI, 2006, 61-76. Zbl 1103.20038 MR 2216701

[Ers04] A. Erschler, Boundary behavior for groups of subexponential growth. Ann. of Math. (2) 160 (2004), 1183-1210. Zbl 1089.20025 MR 2144977

[FG85] J. Fabrykowski and N. Gupta, On groups with sub-exponential growth functions. $J$. Indian Math. Soc. (N.S.) 49 (1985), 249-256. Zbl 0688.20013 MR 942349

[FG91] J. Fabrykowski and N. Gupta, On groups with sub-exponential growth functions. II. J. Indian Math. Soc. (N.S.) 56 (1991), 217-228. Zbl 0868.20029 MR 1153150

[Gri83] R. I. Grigorchuk, On the Milnor problem of group growth. Dokl. Akad. Nauk SSSR 271 (1983), 30-33; English transl. Soviet Math. Dokl. 28 (1983), 23-26. Zbl 0547.20025 MR 712546 
[Gri84] R. I. Grigorchuk, Degrees of growth of finitely generated groups, and the theory of invariant means. Izv. Akad. Nauk SSSR Ser. Mat. 48 (1984), 939-985; English transl. Math. USSR-Izv. f25 (1985), 259-300. Zbl 0583.20023 MR 0764305

[LMR95] P. Longobardi, M. Maj, and A. H. Rhemtulla, Groups with no free subsemigroups. Trans. Amer. Math. Soc. 347 (1995), 1419-1427. Zbl 0833.20043 MR 1277124

[OS96] A. Yu. Ol'shanskii and A. Storozhev, A group variety defined by a semigroup law. J. Austral. Math. Soc. Ser. A 60 (1996), 255-259. Zbl 0859.20022 MR 1375590

Received July 20, 2007; revised July 16, 2008

L. Bartholdi, Mathematisches Institut, Bunsenstraße 3-5, 37073 Göttingen, Germany

E-mail: laurent.bartholdi@gmail.com

F. Pochon, Institut de Mathématiques B (IMB), École Polytechnique Fédérale de Lausanne (EPFL), 1015 Lausanne, Switzerland

E-mail: floriane.pochon@gmail.com 